\title{
Nonlocality in Deuteron Stripping Reactions
}

\author{
N. K. Timofeyuk and R. C. Johnson \\ Department of Physics, University of Surrey, Guildford, Surrey GU2 7XH, United Kingdom
}

(Received 29 November 2012; published 12 March 2013; corrected 13 March 2013)

\begin{abstract}
We propose a new method for the analysis of deuteron stripping reactions, $A(d, p) B$, in which the nonlocality of nucleon-nucleus interactions and three-body degrees of freedom are accounted for in a consistent way. The model deals with equivalent local nucleon potentials taken at an energy shifted by $\sim 40 \mathrm{MeV}$ from the " $E_{d} / 2$ " value frequently used in the analysis of experimental data, where $E_{d}$ is the incident deuteron energy. The " $E_{d} / 2$ " rule lies at the heart of all three-body analyses of $(d, p)$ reactions performed so far with the aim of obtaining nuclear structure properties such as spectroscopic factors and asymptotic normalization coefficients that are crucial for our understanding of nuclear shell evolution in neutron- and proton-rich regions of the nuclear periodic table and for predicting the cross sections of stellar reactions. The large predicted shift arises from the large relative kinetic energy of the neutron and proton in the incident deuteron in those components of the $n+p+A$ wave function that dominate the $(d, p)$ reaction amplitude. The large shift reduces the effective $d-A$ potentials and leads to a change in predicted $(d, p)$ cross sections, thus affecting the interpretation of these reactions in terms of nuclear structure.
\end{abstract}

DOI: 10.1103/PhysRevLett.110.112501

PACS numbers: 25.45.Hi, 21.10.Jx, 27.40.+Z

All practical approaches to the nuclear many-body problem are based on the concept of effective interactions. These are designed to treat a subset of the many-body degrees of freedom explicitly. The implicit degrees of freedom are manifest in a set of parameters that determine the effective interactions. They also give rise to special features of the effective nuclear Hamiltonian. For example, complex optical potentials arise in scattering problems reflecting the possibility of permanent flux loss out of the chosen subspace into the implicit space [1].

Another well-known feature of the optical potential is nonlocality. It arises because the effective interaction takes into account transitions from a point in the explicit space to intermediate points in the implicit space and back again. The initial and final points in the explicit space do not have to have the same spatial coordinates even if some intermediate points conserve energy. For nucleon-nucleus scattering nonlocality has two implications: (i) an equivalent local potential describing this scattering is energy dependent [2] and (ii) the scattering wave function corresponding to the nonlocal potential is smaller in the nuclear interior than the local scattering wave function [3]. These properties of nonlocal potentials are relevant to the application of nuclear reaction theory to the interpretation of experiment.

In this Letter we address the practical implications of nonlocality for the interpretation of the transfer reactions $A(d, p) B$ in terms of nuclear structure. These reactions are at the frontier of the experimental study of shell evolution and changing magic numbers in neutron- and proton-rich regions of the nuclear periodic table, concepts which also have implications for the evolution of the element abundances in the Universe.
In contrast to the case of elastic deuteron scattering, where the neutron and proton are relatively weakly correlated in the loosely bound deuteron and no new nonlocality effects arise over and above those already included in the nucleon optical potentials [4], new effects are expected to arise in the $A(d, p) B$ reaction. Its amplitude depends only on those coherent superpositions of the elastic $(n+p)+A$ and breakup $n+p+A$ components that are taken at the separations between $n$ and $p$ that are comparable to the range of nonlocality of the neutron and proton optical potentials.

The $A(d, p) B$ reaction has the attractive feature of being successfully described in terms of a $n+p+A$ model in which only the degrees of freedom associated with the deuteron are treated explicitly while a nuclear target $A$ is represented by the many-body wave function unaffected by the $n-A$ and $p-A$ interactions [5-7]. A crucial element of these theories is that channels in which the $d-A$ system is excited into its continuum of broken-up states $n+p+A$ have to be included explicitly if the theory is to correctly represent experiment [8]. The most important continuum contributions to this reaction can be accounted for, over a useful range of incident deuteron energies [9], by use of the adiabatic distorted wave approximation (ADWA) [5]. A crucial feature of the ADWA is that it is an approximation to the many-body reaction amplitude in which the manybody nuclear structure is incorporated through a transfer form factor. This allows the ratio of the measured and calculated cross sections to be interpreted in terms of key nuclear structure parameters in a very practical way. The ADWA is also able to include microscopic models of the nucleon optical potentials, but how it should be modified to include nonlocalities has been an open question. 
The need to include nonlocality in the $n+p+A$ description of the $(d, p)$ reaction has been highlighted in Ref. [10] where the cross sections have been calculated from the three-body Faddeev theory. However, this theory neglects the internal structure of $A$ and ignores manybody aspects of the $n+p+A$ wave function so that the ratio of the measured and calculated cross sections cannot be used to give useful nuclear structure information. Reference [11] suggests a way that the missing manybody information can be included into Faddeev equations. However, the result is a formalism that is too complicated for wide use by nuclear experimentalists and, in fact, no applications have been made so far. The new formalism also requires the use of nonlocal nucleon potentials thus emphasizing the need to modify the existing ADWA to include nonlocalities in a simple way.

The standard way of treating $(d, p)$ reactions within the ADWA is to use local nucleon optical potentials at half the incident deuteron energy. For elastic deuteron scattering this idea can be simply understood as being a consequence of the loose binding of the deuteron [4]. For center-of-mass deuteron kinetic energy $E_{d}$ the neutron and proton on the average each have kinetic energy $E_{d} / 2$. The same result can be derived [4] by using a nonlocal model of the $N-A$ potentials with a realistic nonlocality range, i.e., a range that is much smaller than the size of the deuteron. The corresponding nonlocal deuteron potential has a range of nonlocality equal to one-half of the nucleon nonlocality range. We show here that the inclusion of three-body degrees of freedom and nonlocal effects in a consistent way, when applied to the $(d, p)$ reactions, modifies this idea significantly and we suggest a simple way of introducing the necessary modifications while allowing existing nuclear reaction codes to be used.

We assume a three-body $p+n+A$ model where the nucleon- $A$ interactions $V_{N A}$ are nonlocal potentials of the Perey-Buck type [2], i.e., for the $n-A$ case

$$
V_{n A} \Psi\left(\boldsymbol{r}_{n}, \boldsymbol{r}_{p}\right)=\int d \boldsymbol{r}_{n}^{\prime} V_{n A}\left(\boldsymbol{r}_{n}, \boldsymbol{r}_{n}^{\prime}\right) \Psi\left(\boldsymbol{r}_{n}^{\prime}, \boldsymbol{r}_{p}\right),
$$

where

$$
V_{n A}\left(\boldsymbol{r}_{n}, \boldsymbol{r}_{n}^{\prime}\right)=H\left(\boldsymbol{r}_{n}-\boldsymbol{r}_{n}^{\prime}\right) U_{n A}\left(\left(\boldsymbol{r}_{n}+\boldsymbol{r}_{n}^{\prime}\right) / 2\right),
$$

with the Perey-Buck nonlocality factor

$$
H(s)=\pi^{-3 / 2} \beta_{n}^{-3} e^{-\left(s / \beta_{n}\right)^{2}}
$$

with a nonlocality range $\beta_{n}$. Perey and Buck found that the value $\beta_{n}=0.85 \mathrm{fm}$ gave a good account of the energy dependence of $n-A$ scattering known at that time. They also showed that an equivalent local potential $U_{\text {loc }}$ that gave the same $n-A$ scattering phase shifts as $V_{n A}$ is accurately given as the solution of the equation

$$
U_{\mathrm{loc}}=U_{n A} \exp \left[-\frac{M_{n} \beta_{n}^{2}}{2 \hbar^{2}}\left(E-U_{\mathrm{loc}}\right)\right],
$$

where $M_{n}$ is the neutron mass.

The Schrödinger equation for the three-body wave function $\Psi(\boldsymbol{r}, \boldsymbol{R})$, expressed in terms of relative and center-of-mass coordinates of $n$ and $p, \boldsymbol{r}=\boldsymbol{r}_{p}-\boldsymbol{r}_{n}$, $\boldsymbol{R}=\left(\boldsymbol{r}_{n}+\boldsymbol{r}_{p}\right) / 2$, is

$\left(E-T_{r}-T_{R}-V_{n p}(\boldsymbol{r})\right) \Psi(\boldsymbol{r}, \boldsymbol{R})=\left(V_{n A}+V_{p A}\right) \Psi(\boldsymbol{r}, \boldsymbol{R})$,

where $T_{r}\left(T_{R}\right)$ is the kinetic energy operator associated with the coordinate $\boldsymbol{r}(\boldsymbol{R})$ and

$$
\begin{aligned}
V_{n A} \Psi(\boldsymbol{r}, \boldsymbol{R})= & 8 \int d \boldsymbol{R}^{\prime} V_{n A}\left(\boldsymbol{R}-\frac{\boldsymbol{r}}{2}, 2 \boldsymbol{R}^{\prime}-\boldsymbol{R}-\frac{\boldsymbol{r}}{2}\right) \\
& \times \Psi\left(\boldsymbol{r}-2\left(\boldsymbol{R}^{\prime}-\boldsymbol{R}\right), \boldsymbol{R}^{\prime}\right) .
\end{aligned}
$$

The proton term is

$$
\begin{aligned}
V_{p A} \Psi(\boldsymbol{r}, \boldsymbol{R})= & 8 \int d \boldsymbol{R}^{\prime} V_{p A}\left(\boldsymbol{R}+\frac{\boldsymbol{r}}{2}, 2 \boldsymbol{R}^{\prime}-\boldsymbol{R}+\frac{\boldsymbol{r}}{2}\right) \\
& \times \Psi\left(\boldsymbol{r}+2\left(\boldsymbol{R}^{\prime}-\boldsymbol{R}\right), \boldsymbol{R}^{\prime}\right) .
\end{aligned}
$$

These formulae are used in Ref. [4] in the evaluation of the folding potential for $d-A$ elastic scattering with nonlocal nucleon potentials. We ignore $1 / A$ recoil effects for simplicity although it is straightforward to include them.

In the ADWA of Johnson and Soper, as reformulated by Johnson and Tandy [12], the three-body wave function $\Psi(\boldsymbol{r}, \boldsymbol{R})$ is needed in an exact form of the $(d, p)$ transition matrix $T_{d p}$ only in the combination $V_{n p} \Psi(\boldsymbol{r}, \boldsymbol{R})$. This projection is approximated by the first term in an expansion in Weinberg states. The latter are well adapted to the description of $\Psi(\boldsymbol{r}, \boldsymbol{R})$ for the small $n-p$ separations needed for an accurate evaluation of $T_{d p}$. Thus in the ADWA $V_{n p} \Psi$ is replaced by

$$
V_{n p} \Psi(\boldsymbol{r}, \boldsymbol{R}) \approx V_{n p} \phi_{0}(\boldsymbol{r}) \chi(\boldsymbol{R}),
$$

where $\phi_{0}$ is the deuteron ground state wave function. The ADWA neglects all the other Weinberg components of $\Psi(\boldsymbol{r}, \boldsymbol{R})[8,9,12,13]$ so that $\chi(\boldsymbol{R})$ satisfies the equation

$$
\left(E_{d}-T_{R}\right) \chi(\boldsymbol{R})=U_{d A} \chi(\boldsymbol{R}),
$$

where $E_{d}=E-\epsilon_{0}, \epsilon_{0}$ is the deuteron binding energy. The nonlocal operator $U_{d A}$ is given by

$$
U_{d A}=\left\langle\phi_{1}\left|V_{n A}+V_{p A}\right| \phi_{0}\right\rangle,
$$

where $\phi_{1}(\boldsymbol{r})=V_{n p} \phi_{0}(\boldsymbol{r}) /\left\langle\phi_{0}\left|V_{n p}\right| \phi_{0}\right\rangle$. For nonlocal $N-A$ potentials of the Perey-Buck type we obtain 


$$
\begin{aligned}
U_{d A} \chi(\boldsymbol{R})= & \int d \boldsymbol{R}^{\prime} U_{d A}\left(\boldsymbol{R}^{\prime}, \boldsymbol{R}\right) \chi\left(\boldsymbol{R}^{\prime}\right) \\
= & \int d \boldsymbol{s} d \boldsymbol{x}\left[\phi_{1}(\boldsymbol{x}+\boldsymbol{s}) U_{n A}\left(\boldsymbol{R}-\frac{\boldsymbol{x}}{2}\right)\right. \\
& \left.+\phi_{1}(\boldsymbol{x}-\boldsymbol{s}) U_{p A}\left(\boldsymbol{R}+\frac{\boldsymbol{x}}{2}\right)\right] H(\boldsymbol{s}) \phi_{0}(\boldsymbol{x}) \chi\left(\frac{\boldsymbol{s}}{2}+\boldsymbol{R}\right) .
\end{aligned}
$$

A similar expression appears in Ref. [4] for the nonlocal $d-A$ optical potential with the crucial difference that $\phi_{1}$ is replaced by the deuteron ground state wave function $\phi_{0}$ in the elastic case. The evaluation of Eq. (11) therefore involves nucleon momenta introduced by scattering by the short range function $\phi_{1}$ that are significantly bigger than the average values in the deuteron ground state and produce large deviations from the " $E_{d} / 2$ " prescription usually assumed in transfer calculations.

As a first orientation to the evaluation of this effect we expand the form factors $U_{N A}\left(\boldsymbol{R} \pm \frac{\boldsymbol{x}}{2}\right)$ into a Taylor series around $x=0$ keeping the first two term only:

$$
U_{N A}\left(\boldsymbol{R} \pm \frac{\boldsymbol{x}}{2}\right) \approx U_{N A}(\boldsymbol{R}) \pm \frac{1}{2} \boldsymbol{x} \cdot \boldsymbol{\nabla} U_{N A}(\boldsymbol{R}) .
$$

This is justified by the smooth behavior of $U_{N A}(\boldsymbol{R})$ and by the short range of potential $V_{n p}$.

Using the representation $\chi\left(\frac{s}{2}+\boldsymbol{R}\right)=e^{(i / 2) s \boldsymbol{K}} \chi(\boldsymbol{R})$, where $\boldsymbol{K}=\frac{1}{i} \boldsymbol{\nabla}$ is the momentum operator associated with the $n-p$ center-of-mass coordinate $\boldsymbol{R}$, we write Eqs. (9) and (11) as

$$
\begin{aligned}
\left(E_{d}-T_{R}\right) \chi(\boldsymbol{R})= & \left(U_{n A}(R)+U_{p A}(R)\right) \tilde{H}_{0}(K) \chi(\boldsymbol{R}) \\
& -\boldsymbol{\nabla}\left(U_{n A}(R)+U_{p A}(R)\right) \tilde{H}_{1}(K) \nabla \chi(\boldsymbol{R}),
\end{aligned}
$$

where for $\lambda=0,1$,

$$
\begin{aligned}
\tilde{H}_{\lambda}(K)= & \left(-\frac{\partial}{K \partial K}\right)^{\lambda} \int d \boldsymbol{s} d \boldsymbol{x} \exp \left(\frac{\imath \boldsymbol{s} \cdot \boldsymbol{K}}{2}\right) H(s) \\
& \times \boldsymbol{\phi}_{1}^{*}(\boldsymbol{x}+\boldsymbol{s}) \boldsymbol{\phi}_{0}(\boldsymbol{x})\left(\frac{\boldsymbol{s} \cdot \boldsymbol{x}}{\boldsymbol{s}^{2}}\right)^{\lambda} .
\end{aligned}
$$

Note that for spherical $H(s), \phi_{1}, \phi_{0}$, the $\tilde{H}_{\lambda}(K)$ are functions of $K^{2}$. A standard procedure from nuclear reaction and nuclear matter theory [14] replaces Eq. (13) by an equivalent local formulation with a local potential $U_{\text {loc }}^{0}(R)$ that is determined by using the replacement

$$
K^{2}(R)=\frac{2 M_{d}}{\hbar^{2}}\left(E_{d}-U_{\mathrm{loc}}^{0}(R)\right),
$$

where $M_{d}$ is the deuteron mass, in the equation

$$
U_{\mathrm{loc}}^{0}(R)=\left(U_{n A}(R)+U_{p A}(R)\right) \tilde{H}_{0}(K) .
$$

Then the solution of Eq. (13) is the product $\chi(\boldsymbol{R})=$ $f(\boldsymbol{R}) \varphi(\boldsymbol{R})$, where $\varphi$ satisfies the local equation

$$
\begin{aligned}
\left(E_{d}-T_{R}\right) \varphi & =U_{\mathrm{loc}} \varphi \\
& =\left[U_{\mathrm{loc}}^{0}+\frac{\hbar^{2}}{M_{d}}\left(\frac{\nabla f}{f}\right)^{2}-\frac{\hbar^{2}}{2 M_{d}} \frac{\nabla^{2} f}{f}\right] \varphi,
\end{aligned}
$$

and the function $f$ (the Perey factor) is the solution of the first order differential equation

$$
\frac{\nabla f}{f}=-\frac{M_{d}}{\hbar^{2}} \nabla\left(U_{n A}+U_{p A}\right) \tilde{H}_{1}(K)
$$

with the boundary condition $f(R) \rightarrow 1$ at $R \rightarrow \infty$. Thus, if the $N-A$ potentials are nonlocal, a local model exists for the potential in the incident channel of the $A(d, p) B$ reaction that accounts both for these nonlocalities and deuteron breakup into the $p+n+A$ continuum. The new local $d-A$ potential, $U_{\text {loc }}$, can be calculated externally and then read in by existing ADWA computer codes.

The functions $\tilde{H}_{\lambda}(K)$ are readily calculated for any nucleon nonlocality and for any model of the $n-p$ system. However, prior to any calculations, it is instructive to follow an approximate procedure which shows clearly how deviations from the " $E_{d} / 2$ " procedure arise. In Eq. (14) we expand the factor $\phi_{1}^{*}(x+s)$ about $s=0$ retaining terms up to second order. This would be expected to be a useful approach if the function $\phi_{1}$ did not vary rapidly as $s$ varies over the range of the nucleon nonlocality factor $H(s)$. The leading term in this expansion gives

$$
\tilde{H}_{0}(K)=\exp \left(-\left(\beta_{d}^{0} K\right)^{2} / 4\right),
$$

where $\beta_{d}^{0}=\beta_{N} / 2$. It was shown in Ref. [4] that when this result is used to determine $U_{\text {loc }}$ through Eq. (16) the result is precisely the sum of neutron and proton potentials taken at $E_{d} / 2$. Corrections arise because the range of $V_{n p}$ and hence $\phi_{1}$ is comparable to the nucleon nonlocality range $\beta_{N}$. Including the leading correction term gives

$\tilde{H}_{0}(K)=e^{-\left(\beta_{d}^{0} K\right)^{2} / 4}-\frac{M}{\hbar^{2}} \frac{\left\langle T_{n p}\right\rangle}{6} \int d s s^{2} e^{(i s \cdot K) / 2} H(s)$,

where $M$ is the nucleon mass and

$$
\left\langle T_{n p}\right\rangle=\int d \boldsymbol{x} \phi_{1}^{*}(\boldsymbol{x})\left(-\frac{\hbar^{2}}{M} \nabla^{2} \phi_{0}(x)\right)
$$

is a measure of the kinetic energy associated with the $n-p$ separations in deuteron within the range of $\phi_{1}(x)$, i.e., the range of $V_{n p}$. Using the Perey-Buck nonlocality factor $H(s)$ we find

$\tilde{H}_{0}(K) \approx\left(1-\frac{M}{\hbar^{2}}\left(\beta_{d}^{0}\right)^{2}\left\langle T_{n p}\right\rangle\right) \exp \left(-\left(\beta_{d} K\right)^{2} / 4\right)$,

where the new nonlocality range $\beta_{d}$ is

$$
\beta_{d}^{2}=\left(\beta_{N} / 2\right)^{2}\left(1-\frac{2}{3} \frac{M}{\hbar^{2}}\left(\beta_{d}^{0}\right)^{2}\left\langle T_{n p}\right\rangle\right),
$$

where we have treated terms of higher order in $\frac{M}{\hbar^{2}}\left(\beta_{d}^{0}\right)^{2}\left\langle T_{n p}\right\rangle$ only approximately. 
For small values of $\frac{M}{\hbar^{2}}\left(\beta_{d}^{0}\right)^{2}\left\langle T_{n p}\right\rangle$ we thus obtain two consequences of nucleon nonlocality in the three-body model: (i) a change in the nonlocality range from the " $E_{d} / 2$ " value $\beta_{N} / 2$, and (ii) the effect of the renormalization factor in front of the exponential in Eq. (22). Effect (ii) has a simple interpretation as an energy shift. When (22) is used to determine the local equivalent deuteron potential through Eq. (16) we find that to leading order in $\frac{M}{\hbar^{2}}\left(\beta_{d}^{0}\right)^{2}\left\langle T_{n p}\right\rangle$ we must sum the nucleon optical potentials at energy $E_{d} / 2+\Delta E$, where the energy shift

$$
\Delta E=\frac{1}{2}\left\langle T_{n p}\right\rangle
$$

is determined entirely by the properties of $V_{n p}$ and is independent of the precise value of the nucleon nonlocality range to the approximation we have used so far. For the Hulthén potential $V_{n p}(x)=V_{0} /\left(e^{\left(\beta_{H}-\kappa\right) x}-1\right)$, where $\beta_{H}^{-1}$ is a range parameter and $\kappa^{2}=M \epsilon_{0} / \hbar^{2},\left\langle T_{n p}\right\rangle=$ $\frac{\hbar^{2}}{M} \beta_{H}\left(\beta_{H}+2 \kappa\right)$. Using $\beta_{H}=6.255 \kappa$ from Ref. [15] and $\kappa=0.232 \mathrm{fm}^{-1}$ we find $\Delta E=57 \mathrm{MeV}$. This is a large energy shift leading to reduction in depth of the deuteron distorting potential which will affect the ADWA predictions of $(d, p)$ cross sections.

The expansion parameter $\frac{M}{\hbar^{2}}\left(\beta_{d}^{0}\right)^{2}\left\langle T_{n p}\right\rangle$ used above has the value 0.5 for $\beta_{N}=0.85 \mathrm{fm}$ and is not particularly small so that a more careful analysis is needed. Using the Hulthén potential to evaluate $\tilde{H}_{0}(K)$ and $\tilde{H}_{1}(K)$ exactly we find that they can both be represented by single Gaussian functions

$$
\tilde{H}_{\lambda}(K)=\tilde{H}_{\lambda}(0) \exp \left(-\beta_{d}^{2} K^{2} / 4\right) .
$$

For $\beta_{N} / 2=0.425 \mathrm{fm}$ we obtain an effective nonlocality range $\beta_{d}=0.40 \mathrm{fm}$, larger that the value of $0.35 \mathrm{fm}$ predicted by Eq. (23). We also get the energy shift $\Delta E=43 \mathrm{MeV}$ which is $25 \%$ smaller than the estimate based on the second order expansion.

We have evaluated the role of the new " $E_{d} / 2+\Delta E$ " prescription for ${ }^{40} \mathrm{Ca}(d, p){ }^{41} \mathrm{Ca}$ reaction at $E_{d}=11.8 \mathrm{MeV}$, measured in Ref. [16], where the $1 / A$ effects neglected here should be small. An energy-independent phenomenological nonlocal potential for proton $-{ }^{40} \mathrm{Ca}$ scattering is given in Ref. [17]. This potential has a nonlocality range $\beta_{N}=0.97 \mathrm{fm}$, for which $\Delta E=40 \mathrm{MeV}$. The corresponding potential $U_{\mathrm{loc}}^{0}$ in the ${ }^{40} \mathrm{Ca}+d$ channel, shown in Fig. 1(a), has shallower real and imaginary parts than the Johnson-Soper " $E_{d} / 2$ " potential $U_{\mathrm{IS}}$. This change gives larger ${ }^{40} \mathrm{Ca}(d, p){ }^{41} \mathrm{Ca}$ cross sections [see Fig. 1(b), solid line]. The latter were calculated with the TWOFNR code [18] in the zero-range approximation by reading in the $U_{\mathrm{loc}}^{0}$ as the distorting potential in the incident channel and using the same ${ }^{40} \mathrm{Ca}+n$ binding potential as in Ref. [17]. The spectroscopic factor was set equal to one. The nonlocality in the two-body $p-{ }^{41} \mathrm{Ca}$ channel has been taken into account by standard Perey damping of the proton distorting wave. The cross sections increased by $28 \%$ and $17 \%$ for the
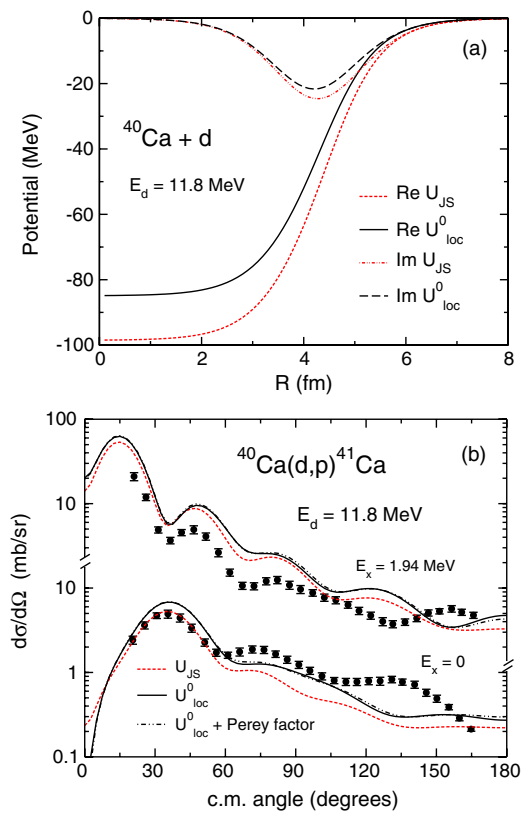

FIG. 1 (color online). (a) Local $U_{\mathrm{loc}}^{0}$ and Johnson-Soper $U_{\mathrm{JS}}$ potentials calculated for ${ }^{40} \mathrm{Ca}+d$ and (b) the ${ }^{40} \mathrm{Ca}(d, p){ }^{41} \mathrm{Ca}$ cross sections obtained with $U_{\mathrm{JS}}$ and $U_{\mathrm{loc}}^{0}$ for the ground and the first excited state in ${ }^{41} \mathrm{Ca}$. The calculations with Perey damping are shown by dot-dashed lines.

ground and the first excited states in ${ }^{41} \mathrm{Ca}$. The corresponding spectroscopic factors obtained from comparison of theoretical and experimental cross sections would be reduced by the same proportions.

We have also calculated the Perey factor $f$ and its effect on the $(d, p)$ cross section. We solved Eq. (18) analytically getting the formula which is very close to the one used in many transfer reaction codes. We have also found that terms containing derivatives of $f$ in the right-hand side of Eq. (17) are small so that $U_{\mathrm{loc}} \approx U_{\mathrm{loc}}^{0}$. Details will be given elsewhere. Thus, the Perey damping of the deuteron distorted wave can also be accounted for in existing transfer reaction codes when $U_{\text {loc }}^{0}$ are read in. In the ${ }^{40} \mathrm{Ca}(d, p)^{41} \mathrm{Ca}$ reaction discussed above the Perey damping has no effect [see Fig. 1(b)].

In conclusion, starting from a three-body $n+p+A$ model with energy-independent nonlocal nucleon optical potentials we have derived a simple local two-body model for the distorting potential in the $d-A$ channel. This model includes equivalent local energy-dependent nucleon potentials taken at an energy shifted with respect to the widely used " $E_{d} / 2$ " rule by a predicted amount determined mainly by the $n-p$ kinetic energy averaged over the range of the $n-p$ potential. Since this range is much smaller than the deuteron radius the associated kinetic energy shift is much larger than what is appropriate for calculating the elastic deuteron potential from local nucleon potentials. Since the depth of nucleon optical potentials decreases with energy the local-equivalent deuteron potential for $(d, p)$ reactions, calculated using the new " $E_{d} / 2+\Delta E "$ prescription, has shallower real and 
absorptive parts in the deuteron channel and in the case discussed here leads to increased theoretical $(d, p)$ cross sections. This increase will affect the interpretation of the $(d, p)$ experiments in terms of nuclear structural quantities such as spectroscopic factors and asymptotic normalization coefficients. These changes may have a substantial effect on the understanding nuclear shell evolution and on the prediction of cross sections for stellar reactions based on these quantities.

Finally, the newly discovered energy shift in $(d, p)$ reactions should be a common feature of many-body reactions involving transfer from an incident two-body projectile bound by a short range potential. Since the dominant mechanism confines the relative motion in the projectile to a small volume within the range of this potential the projectile's constituents gain an additional effective energy because of the uncertainty principle. The precise value of this energy does not depend strongly on the range of nonlocality. This range mainly influences the effective potential in the entrance channel via the energy dependence of the optical potentials of the projectile's constituents.

We gratefully acknowledge the support from U.K. STFC Grant No. ST/J000051/1.

[1] H. Feshbach, Ann. Phys. (N.Y.) 5, 357 (1958).

[2] F. Perey and B. Buck, Nucl. Phys. 32, 353 (1962).
[3] F. Perey, Direct Interactions and Nuclear Reaction Mechanisms (Gordon and Breach, New York, 1963), p. 125.

[4] R. C. Johnson and P. J. R. Soper, Nucl. Phys. A182, 619 (1972).

[5] R. C. Johnson and P. J. R. Soper, Phys. Rev. C 1, 976 (1970).

[6] G. Rawitscher, Nucl. Phys. A241, 365 (1975).

[7] N. Austern, Y. Iseri, M. Kamimura, M. Kawai, G. Rawitscher, and M. Yahiro, Phys. Rep. 154, 125 (1987).

[8] R. C. Johnson, AIP Conf. Proc. 791, 128 (2005).

[9] F. M. Nunes and A. Deltuva, Phys. Rev. C 84, 034607 (2011).

[10] A. Deltuva, Phys. Rev. C 79, 021602 (2009).

[11] A. M. Mukhamedzhanov, V. Eremenko, and A. I. Sattarov, Phys. Rev. C 86, 034001 (2012).

[12] R. C. Johnson and P. C. Tandy, Nucl. Phys. A235, 56 (1974).

[13] A. Laid, J. A. Tostevin, and R. C. Johnson, Phys. Rev. C 48, 1307 (1993).

[14] G. R. Satchler, Direct Nuclear Reactions (Oxford Press, New York, 1983), pp. 819-824.

[15] Y. Yamaguchi, Phys. Rev. 95, 1628 (1954).

[16] U. Schmidt, R. Stock, and P. Turek, Nucl. Phys. 53, 77 (1964).

[17] M. M. Gianinni and G. Ricco, Ann. Phys. (N.Y.) 102, 458 (1976).

[18] J.A. Tostevin, University of Surrey version of the code TWOFNR (of M. Toyama, M. Igarashi, and N. Kishida), http://www.nucleartheory.net/NPG/code.htm. 